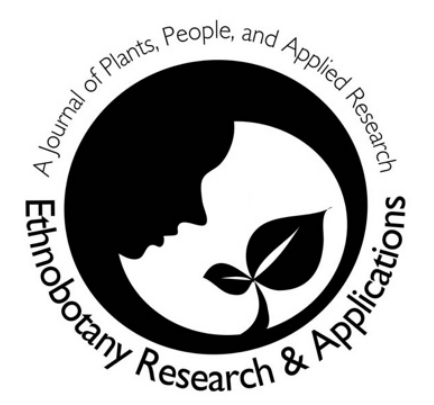

\title{
Ethnobiology of Mountain Communities in Asia -A Book Review
}

Zubair A. Malik

Correspondence

Zubair A. Malik ${ }^{1,2}$

${ }^{1}$ Department of Botany and Microbiology, HNB Garhwal University, Srinagar Garhwal Uttarakhand (India)-246174

2 Presently at Department of Biology, Govt. HSS Harduturoo Anantnag (J\&K) - 192201

*Corresponding Author: malikmzubair081@gmail.com

Ethnobotany Research and Applications 23:14 (2022)

\section{Book Review}

Ethnobiology of Mountain Communities in Asia, Abbasi, A. M., \& Bussmann, R. W. (Eds.). (2021) Springer Nature Switzerland. xiv + 439 pp. (Hardcover). EUR 149.99. ISBN: 978-3-030-55493-4; DOI: https://doi.org/10.1007/9783-030-55494-1

The book under review (Ethnobiology of Mountain Communities in Asia) is a part of the book series, Ethnobiology. So far, twelve volumes of this series have been published since 2015 (https://www.springer.com/series/11551). Ethnobiology is the study of the dynamic relationship between plants, animals, people, and the environment. Contemporary ethnobiological research is grounded in respect for all cultures, embracing the principles of prior informed consent, benefit sharing, and general mindfulness.

With about 440 pages and edited by Arshad Mehmood Abbasi and Rainer W. Bussmann, well known in the field of ethnobotany, the book (Ethnobiology of Mountain Communities in Asia), is conceptualized on the fact that plants provide humankind with most of the basic resources, including food and medicine. At present, when human populations have increased that have access to markets, overharvesting of commercially important medicinal species often is the consequence. At the same time, habitat loss, climate change, and invasive species further threaten wild plant populations, while traditional knowledge associated with plant use is being eroded by urbanization. Thus, never before in human history has there been a greater need to discover, understand, conserve, and sustainably use culturally important plant resources.

The book contains 19 chapters on the ethnobiology of mountain communities of a few Asian countries (Bhutan, India, Nepal, Pakistan, Sri Lanka, and Thailand). The ethnobiological studies presented are however not evenly distributed among the different regions of Asia. Out of the total 19 chapters, 13 (69\%) deal with ethnobiology of Pakistan while the remaining 6 chapters (31\%) present different aspects of ethnobotany (rather than ethnobiology) of Sri Lanka (2 Chapters), Bhutan (1), Thailand (1), Nepal (1) and India (1). Ethnobotanical and ethno-zoological studies are not also distributed evenly. Out of the 19 chapters, only three chapters (16\%) present ethno-zoological studies (from Pakistan only). Similar case studies from other regions are missing. Out of three ethno-zoological chapters, only one chapter (Ch 09, Ethno-Medicinal Uses of Wildlife in Azad Jammu and Kashmir, Pakistan, P175) has provided detailed morphological descriptions of the animal species along with their distribution, local name, common name, parts used, and diseases cured. In other two chapters (Ch 11, P. 221 and Ch 14, P 269) this taxonomically important information is missing. On P 118 and 119 some important medicinal plants from 'Ayubia National Park, Pakistan' are presented without names. The botanical description of these plants along with their names on the photo plates could help in their identification. Certain instances indicate poor proofreading of this 
book (e.g., at P 52 a sentence is presented as: A total of 105, 105,105 wild vegetables, belonging to 888,888 genera and 505,050 families, were cited by the informants). Also, the book is very expensive for personal collection (price = EUR 149.99 that is more than 12K Indian Rupees, 20K Nepalese Rupees and 34K Sri Lankan Rupees).

Despite the above-mentioned shortcomings (which can be improved in the next editions/volumes), the book provides baseline information/data for further studies in these regions. The included index of plant species at the end of book is appreciable. The plant species are arranged in an alphabetical order which will help the reader to locate a particular plant species immediately. The recommendations (regarding the conservation/multiplication of medicinal plants) contained in this book, if implemented properly and followed sincerely, will surely help in their conservation and management. The present volume is expected to provide an in-depth introduction to the ethnobiological knowledge of the peoples of Asian mountain regions, with information both from a historic perspective and data from the most recent scientific studies in the region, including the most up-to-date literature sources. I hope that the present book will give both interested laypeople and professionals an opportunity to learn about the fascinating biodiversity and ethnobiology of Asian mountain regions and will spark interest in its further documentation, sustainable use, and conservation. 